Full length article

\title{
Increased gait variability may not imply impaired stride-to-stride control of walking in healthy older adults
}

\author{
Winner: 2013 Gait and Clinical Movement Analysis Society Best Paper Award \\ Jonathan B. Dingwell ${ }^{\mathrm{a}, *, 1}$, Mandy M. Salinas ${ }^{\mathrm{a}}$, Joseph P. Cusumano ${ }^{\mathrm{b}}$ \\ ${ }^{a}$ Department of Kinesiology E Health Education, University of Texas, Austin, TX 78712, United States \\ ${ }^{\mathrm{b}}$ Department of Engineering Science E Mechanics, Pennsylvania State University, University Park, PA 16802, United States
}

\section{A R T I C L E I N F O}

\section{Article history:}

Received 18 June 2016

Received in revised form 12 February 2017

Accepted 18 March 2017

\section{Keywords:}

Walking

Stepping

Variability

Older adults

Motor control

\begin{abstract}
A B S T R A C T
Older adults exhibit increased gait variability that is associated with fall history and predicts future falls. It is not known to what extent this increased variability results from increased physiological noise versus a decreased ability to regulate walking movements. To "walk", a person must move a finite distance in finite time, making stride length $\left(L_{n}\right)$ and time $\left(T_{n}\right)$ the fundamental stride variables to define forward walking. Multiple age-related physiological changes increase neuromotor noise, increasing gait variability. If older adults also alter how they regulate their stride variables, this could further exacerbate that variability. We previously developed a Goal Equivalent Manifold (GEM) computational framework specifically to separate these causes of variability. Here, we apply this framework to identify how both young and high-functioning healthy older adults regulate stepping from each stride to the next. Healthy older adults exhibited increased gait variability, independent of walking speed. However, despite this, these healthy older adults also concurrently exhibited no differences (all $p>0.50$ ) from young adults either in how their stride variability was distributed relative to the GEM or in how they regulated, from stride to stride, either their basic stepping variables or deviations relative to the GEM. Using a validated computational model, we found these experimental findings were consistent with increased gait variability arising solely from increased neuromotor noise, and not from changes in stride-to-stride control. Thus, age-related increased gait variability likely precedes impaired stepping control. This suggests these changes may in turn precede increased fall risk.
\end{abstract}

(c) 2017 Elsevier B.V. All rights reserved.

\section{Introduction}

Falls pose a significant [1,2] and extremely costly [3] health problem for older adults. Falls are the leading cause of both fatal and nonfatal injuries in older adults [4], yielding extremely high injury rates and severity [4,5]. Most falls occur during whole-body movements like walking $[1,6]$. Older adults exhibit increased gait variability [7] that is associated with fall history [8] and predicts future falls [9]. However, it is not yet understood what drives this increased variability. In particular, it is not known to what extent this increased variability results from increased physiological

\footnotetext{
* Corresponding author at: Department of Kinesiology \& Health Education, The University of Texas at Austin, 2109 San Jacinto Blvd., Stop D3700 Austin, TX 78712-1415, United States.

E-mail address: jdingwell@austin.utexas.edu (J.B. Dingwell).

1 web: http://www.edb.utexas.edu/khe/nbl/.
}

noise on the one hand, or a decreased ability to control (i.e., to regulate) the effects of this increased physiological noise on the other.

Neuromotor noise increases with age [10-12]. The number of spinal motor neurons decreases, as do both the number and size of muscle fibers (sarcopenia) [10,13], yielding more discretized force generation. Muscle spindle and proprioceptor function deteriorates, degrading sensory information accuracy [11]. Nerve conduction velocities decrease [11], increasing time delays in sensory-motor feedback loops. Age-related increases in neuromotor noise contribute to impaired mechanical performance of muscle [13] and likely also to increased gait variability [14]. Higher-level cognitive impairments also contribute to changes in gait variability $[15,16]$. What is not known is the extent to which these physiological changes might be compensated for by the adaptability of motor regulation in the central nervous system. 
Our previous work demonstrated that healthy older adults exhibit increased gait variability [7], increased local dynamic instability [17], and increased variability of muscle activations [14] across walking speeds. Subsequent numerical studies using a 3D dynamic walking model [18] supported the possibility that increased neuromotor noise may directly influence both kinematic gait variability and fall risk. However, in that model, increases in probability of falling only occurred with sufficiently large simulated motor noise levels [18]. Likewise, global measures of variability and/or local dynamic stability (that compute averages over many strides) cannot reveal where this variability arises from on a stride-by-stride basis. Gait variability reflects the summed effects of both small disturbances (i.e., noise) experienced while walking and the neural control actions taken to respond to them. What is needed is a means to separate these two influences.

Complicating this question is equifinality [19-22]: for most real tasks, there exists an infinite number of movement solutions that equally satisfy the task goal. Thus, certain control actions may reduce variability of some variables, while simultaneously increasing variability of others. We recently developed a computational framework for determining how both noise and stride-to-stride control actions in walking contribute to gait variability [23,24]. To "walk" a person must move a finite distance (i.e., take a step) in finite time. Neural commands generate muscle forces that move the legs to enact stepping movements. These movements yield stride variables of stride length $\left(L_{n}\right)$ and time $\left(T_{n}\right)$, which then define the minimal set of task variables at the end-effector level needed to fully describe task performance in walking [23,24]. For treadmill walking, an infinite number of possible strategies exist that can successfully achieve this task [23,24]. Among these infinite options, healthy adults choose to allow fluctuations in stride length $\left(L_{n}\right)$ and time $\left(T_{n}\right)$ to go uncorrected over multiple consecutive strides, but directly correct deviations in stride speed $\left(S_{n}=L_{n} / T_{n}\right)$. This implies the existence of a "Goal Equivalent Manifold" (GEM) [19,21] where all combinations of $\left[T_{n}, L_{n}\right]$ that achieve the same $S_{n}$ equally satisfy the goal to maintain constant walking speed (Fig. 2A). We then analyze not only the magnitude of the variability observed, but also quantify the degree to which fluctuations about the GEM on any given stride are directly corrected for on subsequent strides [24,25]. This fundamentally dynamical analysis of movement fluctuations near the GEM yields a quantification of the "strength" of motor regulation (i.e., control) that is independent of the amount of variability present in the data [21,22]. Our models of stride-to-stride fluctuation dynamics were validated experimentally [24,25] and our overall theoretical predictions replicated in at least three independent studies [26-28].

Age-related changes in neuromotor noise might contribute directly to increased movement variability, but might also (separately) cause people to change how they regulate (from stride-to-stride) their stride variables. If so, the GEM framework and our GEM-based dynamical analyses would capture this by allowing an explicit separation of the effects of noise from those of control. Indeed, one recent study applied our analyses to older adults [26]. However, their findings might be attributable to differences in physiological noise, to differences in control, or possibly to differences in walking speed. Conversely, if otherwise healthy older adults can accommodate modest increases in neuromuscular noise, as suggested by our modeling work [18], then increased variability might not induce concomitant changes in how older adults regulate their stride variables and/or stride speed from each stride to the next. Here, we directly tested these competing predictions by applying our GEM-based analyses [24] to ascertain how healthy young and older adults [7,14,17] regulate their stepping variables from stride to stride, particularly with respect to maintaining stride speed $[23,24]$.

\section{Methods}

\subsection{Participants}

Data from a previous study $[7,14,17]$ were analyzed. Participants included 17 young $(\mathrm{YH})$ and 17 older $(\mathrm{OH})$ healthy adults, matched between groups for age, height, and body mass (Table 1). All participants were free from any injuries, disabilities, gait asymmetries, or medications that might have affected their walking. All participants signed institutionally approved informed consent forms prior to participating.

\subsection{Experimental protocol}

Participants walked on a level treadmill (Woodway Desmo S, Woodway USA, Waukesha WI) while wearing a safety harness (Protecta International, Houston TX). Each participant completed two 5-min walking trials, in pseudo-randomized order [7], at each of 5 speeds ranging from $80 \%$ to $120 \%$ times their preferred walking speed, PWS [29]. Participants were instructed to look straight ahead and avoid extraneous movements. Participants rested at least 2 min between trials to minimize fatigue.

Kinematic data were recorded using an 8-camera Vicon system (Oxford Metrics, Oxford, UK) and included data from five markers placed on each foot [7]. Data from one trial from each of 4 young and 2 older subjects were discarded due to poor data quality. Individual heel strikes were defined as the point in each gait cycle where the heel marker of the forward foot was at its most forward point within a gait cycle $[7,24]$. For each stride, $n$, stride time $\left(T_{n}\right)$ was taken as the time between consecutive right heel strikes. Stride length $\left(L_{n}\right)$ was taken as the anterior-posterior distance travelled during each stride. Stride speed $\left(S_{n}\right)$ was then calculated as $S_{n}=L_{n} / T_{n}$. For each trial, we thus obtained full time series of $T_{n}$, $L_{n}$, and $S_{n}$ across all strides in that trial.

\subsection{Data analyses}

For each trial, means and standard deviations of each time series $\left(T_{n}, L_{n}\right.$, and $\left.S_{n}\right)$ were calculated. These data were subjected to a two-factor (Group $\times$ Speed) repeated measures general linear model analysis of variance (ANOVA). All statistical analyses were conducted using Minitab 17 (Minitab, Inc., State College, PA).

To analyze walking dynamics relative to the GEM (Fig. 2A), we first non-dimensionalized each $T_{n}$ and $L_{n}$ time series to unit variance $(\sigma=1)$ to provide an intuitive reference for comparison [24]:

$\tilde{T}_{n}=T_{n} / \sigma\left(T_{n}\right)$ and $\tilde{L}_{n}=L_{n} / \sigma\left(L_{n}\right)$.

This yielded a GEM now defined by the dimensionless walking speed: $\tilde{v}=\operatorname{mean}\left(\tilde{L}_{n} / \tilde{T}_{n}\right)$ with a mean "preferred operating point" on the GEM of: $\left[\tilde{T}^{*}, \tilde{L}^{*}\right]=\left[\operatorname{mean}\left(\tilde{T}_{n}\right), \tilde{v} \tilde{T}^{*}\right]$. We then defined new

Table 1

Participant characteristics (mean $\pm S D$ ), including gender ratio $(M / F)$, age, height, body mass, body mass index, and preferred treadmill walking speeds used to set the treadmill speeds as each subject walked. P-values indicate between-group comparisons from single-factor ANOVA (i.e., un-paired $t$-test).

\begin{tabular}{llll}
\hline & Young Adults & Older Adults & p-value \\
\hline Gender $(\mathrm{M} / \mathrm{F})$ & $12 / 5$ & $11 / 6$ & \\
Age (years) & $23.29 \pm 2.66$ & $72.29 \pm 6.10$ & $3.7 \times 10^{-25}$ \\
Height $(\mathrm{m})$ & $1.733 \pm 0.094$ & $1.694 \pm 0.101$ & 0.253 \\
Body Mass $(\mathrm{kg})$ & $71.11 \pm 9.86$ & $72.29 \pm 12.13$ & 0.757 \\
Body Mass Index $\left(\mathrm{kg} / \mathrm{m}^{2}\right)$ & $23.57 \pm 1.72$ & $25.16 \pm 3.36$ & 0.092 \\
Preferred Walking Speed $(\mathrm{m} / \mathrm{s})$ & $1.296 \pm 0.100$ & $1.299 \pm 0.148$ & 0.952 \\
\hline
\end{tabular}



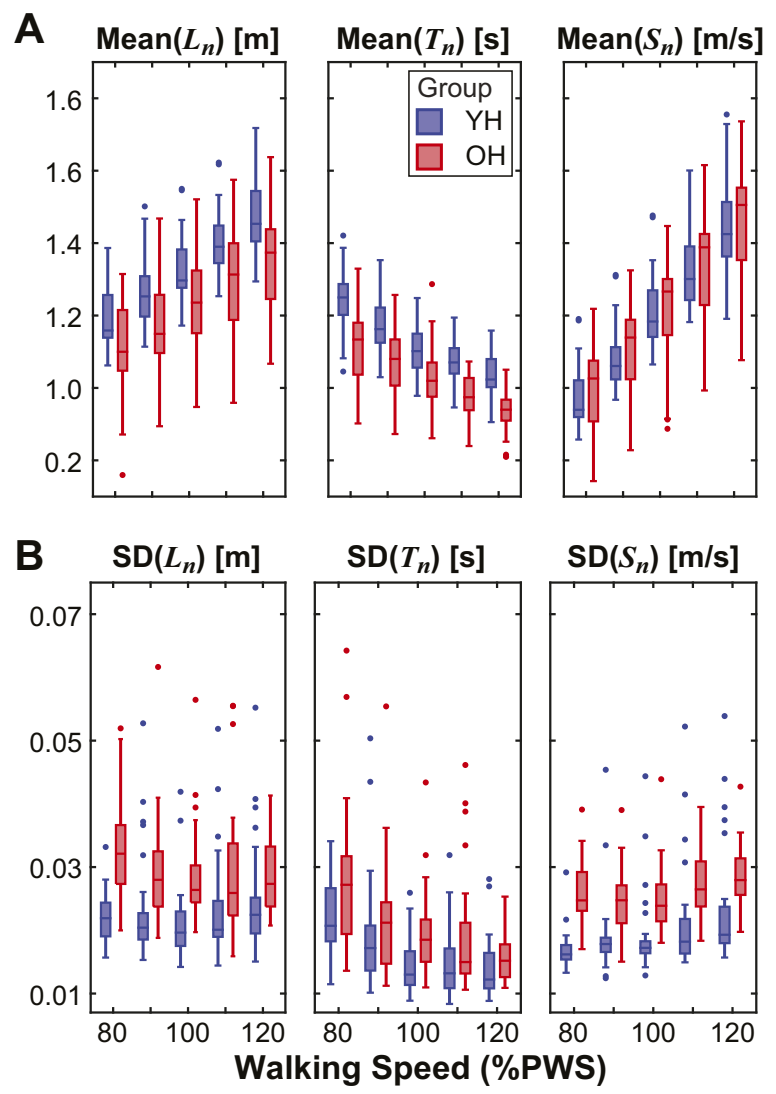

Fig. 1. Basic Stride Parameters: $\boldsymbol{A}$ : Box plots for stride length $\left(L_{n}\right)$, time $\left(T_{n}\right)$, and speed $\left(S_{n}\right)$ for both young $(\mathrm{YH})$ and older $(\mathrm{OH})$ healthy adults walking at all 5 speeds ( $80 \%$ to $120 \%$ of preferred walking speed; PWS). Main effects for walking speed were all highly significant (all $\left.\mathrm{p}_{\mathrm{Spd}}<0.001\right)$. Older $(\mathrm{OH})$ participants took shorter $\left(L_{n}\right.$; $\left.\mathrm{p}_{\mathrm{Grp}}=0.021\right)$ and faster $\left(T_{n} ; \mathrm{p}_{\mathrm{Grp}}<0.001\right)$ strides than $\mathrm{YH}$, but walked at the same speeds $\left(S_{n} ; \mathrm{p}_{\mathrm{Grp}}=0.569\right)$. B: Box plots for within-trial standard deviations for $L_{n}, T_{n}$ and $S_{n}$ for both $\mathrm{YH}$ and $\mathrm{OH}$ groups walking at all 5 speeds. Significant differences between speeds were exhibited for $L_{n}\left(\mathrm{p}_{\mathrm{Spd}}=0.022\right), T_{n}\left(\mathrm{p}_{\mathrm{spd}}<0.001\right)$, and $S_{n}\left(\mathrm{p}_{\mathrm{spd}}\right.$ $<0.001)$. $\mathrm{OH}$ participants exhibited significantly greater variability than $\mathrm{YH}$ for all parameters: $L_{n}\left(\mathrm{p}_{\mathrm{Grp}}=0.001\right), T_{n}\left(\mathrm{p}_{\mathrm{Grp}}=0.036\right)$, and $S_{n}\left(\mathrm{p}_{\mathrm{Grp}}<0.001\right)$. In all plots, each box plot indicates median and inter-quartile range (IQR), each whisker is $1.5 \times \mathrm{IQR}$ in length, and individual symbols $(\bullet)$ indicate data points lying outside this range.

coordinates centered at this operating point, $\tilde{T}_{n}^{\prime}=\tilde{T}_{n}-\tilde{T}^{*}$ and $\tilde{L}_{n}^{\prime}=\tilde{L}_{n}-\tilde{L}^{*}$ and performed a linear coordinate transformation to define deviations either along $\left(\delta_{T}\right)$ or perpendicular to $\left(\delta_{P}\right)$ the GEM as (Fig. 2A):

$\left[\begin{array}{l}\delta_{T} \\ \delta_{P}\end{array}\right]=\frac{1}{\sqrt{1+\tilde{v}^{2}}}\left[\begin{array}{cc}1 & \tilde{v} \\ -\tilde{v} & 1\end{array}\right]\left[\begin{array}{c}\tilde{T}_{n}^{\prime} \\ \tilde{L}_{n}^{\prime}\end{array}\right]$

With corresponding unit vectors (Fig. 2A) defined as:

$\hat{e}_{T}=\frac{1}{\sqrt{1+\tilde{v}^{2}}}\left[\begin{array}{l}\tilde{v} \\ 1\end{array}\right]$ and $\hat{e}_{P}=\frac{1}{\sqrt{1+\tilde{v}^{2}}}\left[\begin{array}{c}-1 \\ \tilde{v}\end{array}\right]$

To quantify how variance was distributed relative to the GEM, the standard deviation of each of these new time series $\left(\delta_{T}\right.$ and $\left.\delta_{P}\right)$ was calculated for each trial. Because data were first normalized to unit variance along both the $L_{n}$ and $T_{n}$ axes, these variability measures reflected not the magnitude of variance, but instead the relative distribution of the variance along the tangent and perpendicular axes. These data were subjected to a three-factor $\left(\right.$ Group $\times$ Speed $\times$ Direction $\left(\hat{e}_{T}\right.$ vs. $\left.\hat{e}_{P}\right)$ repeated measures general linear model ANOVA. We hypothesized subjects would distribute more relative variance tangent to the GEM (i.e., $\sigma\left(\delta_{T}\right)>1$ ), while constraining relative variance perpendicular to the GEM (i.e., $\left.\sigma\left(\delta_{P}\right)<1\right)$. If our $\mathrm{OH}$ adults made more and/or larger errors, on average, with respect to achieving the speed goal, they would exhibit less relative $\sigma\left(\delta_{T}\right)$ and more relative $\sigma\left(\delta_{P}\right)$ compared to $\mathrm{YH}$ adults, as in [26].

However, measures of variability only quantify the average magnitude of differences across all strides. To quantify how deviations in stride variables are regulated from each stride to the next, we need analytical tools that directly quantify the degree and direction of stride-to-stride changes in each variable [22,25]. Here, we computed scaling exponents, $\alpha$, using Detrended Fluctuation Analysis (DFA) [25,30] (see Fig. 3A). $\alpha>1 / 2$ indicates statistical persistence: deviations in one direction are more likely to be followed by deviations in the same direction. $\alpha<1 / 2$ indicates antipersistence: deviations in one direction are more likely to be followed by deviations in the opposite direction. $\alpha=1 / 2$ indicates uncorrelated data: all deviations are equally likely to be followed by deviations in either direction. Consistent with our computational control model results $[21,22,24,25]$, we interpret statistical persistence $(\alpha>1 / 2)$ to indicate variables that are weakly regulated. Variables that are tightly regulated are expected to exhibit either uncorrelated or anti-persistent fluctuations $(\alpha \leq \sim 1 / 2)$. We hypothesized subjects would tightly regulate fluctuations in speed (i.e., $\left.\alpha\left(S_{n}\right) \approx \alpha\left(\delta_{P}\right) \approx 1 / 2\right)$, while allowing fluctuations in other variables to persist (i.e., $\left.\left\{\alpha\left(T_{n}\right), \alpha\left(L_{n}\right), \alpha\left(\delta_{T}\right)\right\}>>^{1} / 2\right)$. If the greater variability $[7,17]$ exhibited by $\mathrm{OH}$ adults resulted from changes in how they regulated their stride variables from stride-to-stride, we would expect differences in $\alpha\left(\delta_{P}\right)$ and/or $\alpha\left(\delta_{T}\right)$ between the two groups.

\subsection{Modeling}

To identify potential mechanisms to explain our experimental findings, we adapted our previously developed model for treadmill walking [24]. In brief, we modeled the dynamics of stride-to-stride fluctuations in stride time and length, $\left[T_{n}, L_{n}\right]$ (Fig. 2A), as a discrete map from each stride to the next:

$\mathbf{x}_{n+1}=\mathbf{x}_{n}+G(I+N) \mathbf{u}\left(\mathbf{x}_{n}\right)+\boldsymbol{\eta}$

where $\mathbf{x}_{n+1}=\left[T_{n+1}, L_{n+1}\right]^{T}$ and $\mathbf{x}_{n}=\left[T_{n}, L_{n}\right]^{T}$ current $(n)$ strides, respectively. $I$ was the $2 \times 2$ identity matrix, $\mathbf{u}\left(\mathbf{x}_{n}\right)=\left[u_{1}, u_{2}\right]^{T}$ was a vector of control inputs, and:

$G=\left[\begin{array}{cc}g_{1} & 0 \\ 0 & g_{2}\end{array}\right], N=\left[\begin{array}{cc}\sigma_{N 1} \varepsilon_{N 1} & 0 \\ 0 & \sigma_{N 2} \varepsilon_{N 2}\end{array}\right]$, and $\boldsymbol{\eta}=\left[\begin{array}{c}\sigma_{\eta 1} \varepsilon_{\eta 1} \\ \sigma_{\eta 2} \varepsilon_{\eta 2}\end{array}\right]$.

Here, $G$ was a matrix of additional gains, $N$ denoted multiplicative noise, and $\boldsymbol{\eta}$ denoted additive noise. Each noise $\sigma_{\mathrm{x}} \varepsilon_{\mathrm{x}}$ term, where $x \in\{N 1, N 2, \eta 1, \eta 2\}$, was composed of an independent Gaussian random variable with zero mean and unit variance $\left(\varepsilon_{x}\right)$, scaled by its corresponding standard deviation $\left(\sigma_{x}\right)$ [24]. Thus, Eq. (4) models each new stride, $\mathbf{x}_{\mathrm{n}+1}$, as arising from the previous stride, $\mathbf{x}_{n}$, modified by some control input, $\mathbf{u}\left(\mathrm{x}_{n}\right)$, that is then influenced by both multiplicative (i.e., signal-dependent $[10,12,31]$ ) and additive noise.

Control inputs, $\mathbf{u}\left(\mathrm{x}_{n}=\left[u_{1}, u_{2}\right]^{T}\right.$, were obtained analytically [24] by deriving an unbiased stochastic optimal single-step controller, based on the Minimum Intervention Principle [20], subject to the expected value of the following cost function:

$C=\alpha e^{2}+\beta p^{2}+\gamma u_{1}^{2}+\delta u_{2}^{2}$ 

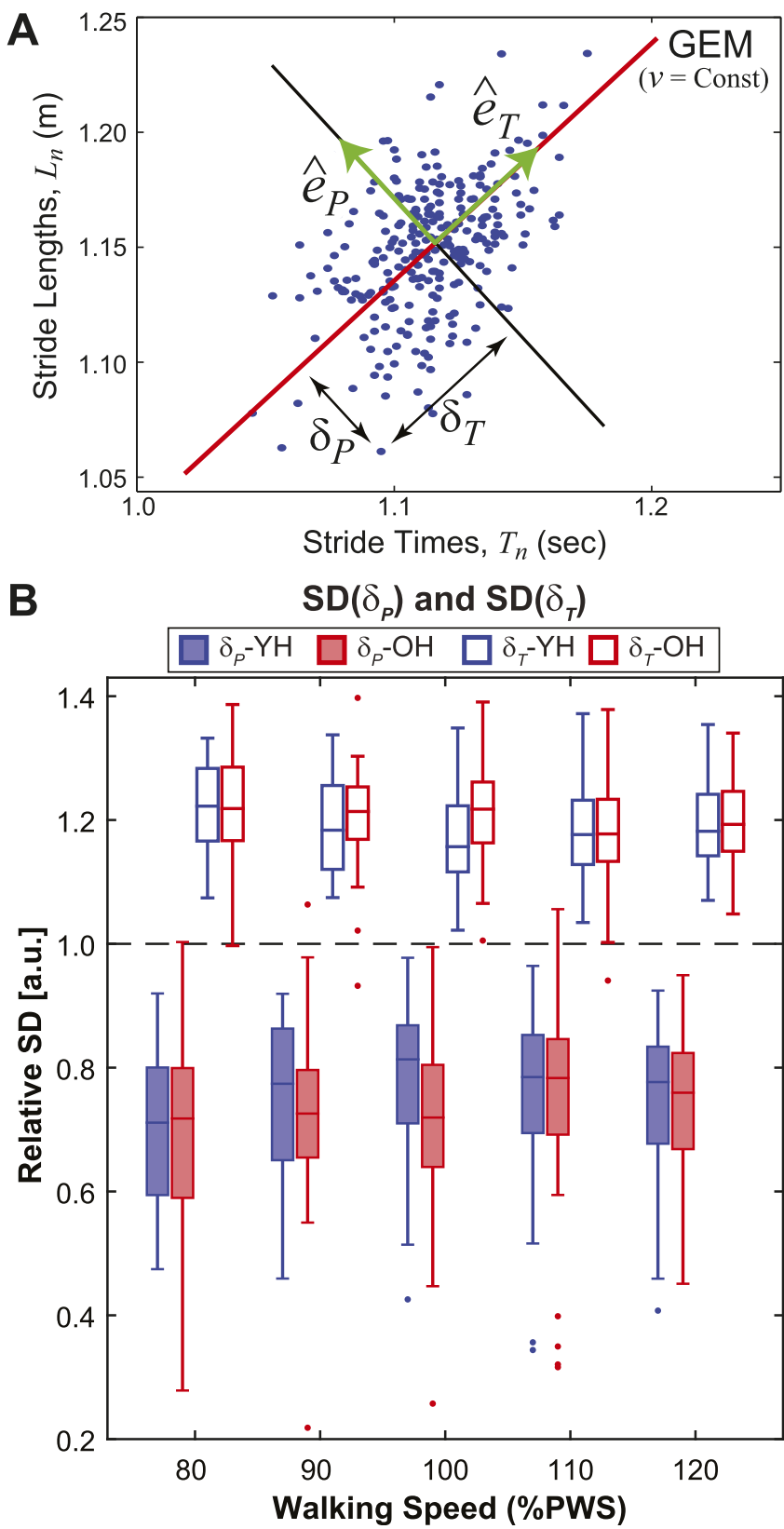

Fig. 2. Variability Relative to the GEM: $\boldsymbol{A}$ : Schematic depiction of the definition of the Goal-Equivalent Manifold (GEM) for maintaining constant walking speed $(v)$, shown in original units. All $[T, L]$ combinations that lie exactly on the GEM (diagonal red line) achieve the exact same speed and thus equally satisfy the goal. Deviations tangent $\left(\delta_{T}\right)$ and perpendicular $\left(\delta_{P}\right)$ to the GEM (Eq. (2)) are indicated for one representative data point. Fluctuations in $\delta_{T}$ that act along the GEM have no effect on achieving the goal to maintain constant speed. Fluctuations in $\delta_{P}$ that act perpendicular to the GEM do directly affect the goal and introduce errors with respect to maintaining constant speed. Normalizing $L_{n}$ and $T_{n}$ each to unit variance changes the numeric values on the axes, but does not change how the data are structured with respect to each other in the $[T, L]$ plane. B: Box plots of within-trial normalized standard deviations for $\delta_{T}$ (open boxes) and $\delta_{P}$ (filled boxes) for both $\mathrm{YH}$ and $\mathrm{OH}$ groups walking at all 5 speeds. Differences between $\delta_{T}$ and $\delta_{P}$ directions were highly statistically significant $\left(\mathrm{p}_{\text {Dir }}<<0.0001\right)$. However, there were no significant differences either across speeds $\left(\mathrm{p}_{\mathrm{Spd}}=0.970\right.$ ), or between $\mathrm{YH}$ and $\mathrm{OH}$ adults $\left(\mathrm{p}_{\mathrm{Grp}}=0.520\right)$. These box plots were constructed in the same manner as in Fig. 1. (For interpretation of the references to colour in this figure legend, the reader is referred to the web version of this article.)

where $e$ is the error with respect to the GEM, $e=L_{n} / T_{n}-v$, and $p$ is the Euclidean distance from the preferred operating point [24]. In Eq. (4), these stochastically optimal control inputs could be tuned away from optimality (with respect to the cost function; Eq. (5)) by changing the gain $(G)$. Setting $g_{1,2} \neq 1$ yields control that is then sub-optimal in minimizing errors with respect to the task goal: i.e., $g_{1,2}<1$ under-corrects goal-relevant errors, while $g_{1,2}>1$ overcorrects goal-relevant errors [24].

Here, we used this model to test how independently varying this gain, $G$, and the noise terms, $N$ and $\eta$, would affect the dynamics (variability and DFA $\alpha$ ) of the relevant stride variables $\left(L_{n}, T_{n}, S_{n}, \delta_{T}\right.$, and $\left.\delta_{P}\right)$. To define baseline parameter values, we took those that approximated an average $\mathrm{YH}$ adult walking at $v=1.21 \mathrm{~m} /$ $\mathrm{s}$, the mean stride speed (i.e., $\operatorname{Mean}\left(S_{n}\right)$ in Fig. $1 \mathrm{~A}$ ) exhibited by our $\mathrm{YH}$ subjects as they walked at their PWS [24]. These baseline parameters were previously determined to be $\sigma_{N 1}=\sigma_{\eta 1}=0.017$ and $\sigma_{N 2}=\sigma_{\eta 2}=0.010$ for Eq. (4) and in Eq. (5), we set $\beta=2.79$ and $\alpha=\gamma=\delta=10$ [24]. To simulate effects of changes in additive noise, we assumed optimal control $\left(g_{1}=g_{2}=1\right)$, kept all other parameters unchanged, and increased the variance of the additive noise terms proportionally from "baseline" $\left(\sigma_{\eta 1}=0.017, \sigma_{\eta 2}=0.010\right)$ to "medium" $\left(\sigma_{\eta 1}=0.0425, \sigma_{\eta 2}=0.025\right)$ to "high" $\left(\sigma_{\eta 1}=0.085, \sigma_{\eta 2}=0.050\right)$. To simulate effects of changes in multiplicative noise, we again assumed optimal control $\left(g_{1,2}=1\right)$, kept all other parameters unchanged, and increased the variance of the multiplicative noise terms proportionally from "baseline" $\left(\sigma_{N 1}=0.017, \sigma_{N 2}=0.010\right)$ to "medium" $\left(\sigma_{N 1}=0.0425, \sigma_{N 2}=0.025\right)$ to "high" $\left(\sigma_{N 1}=0.085, \sigma\right.$ $N_{2}=0.050$ ). To simulate effects of changing controller gains, we assumed "baseline" noise $\left(\sigma_{N 1}=\sigma_{\eta 1}=0.017\right.$ and $\left.\sigma_{N 2}=\sigma_{\eta 2}=0.010\right)$, kept all other parameters unchanged, and increased the controller gains from $g_{1,2}=0.75$ (under-correcting) to $g_{1,2}=1.00$ (optimal) to $g_{1,2}=1.50$ (over-correcting).

For each set of parameter values, we used Eq. (4) to simulate 100 walking trials of 1000 strides each and extracted the same dependent measures as from our experimental data. If age-related changes in neuromotor noise cause older adults to change how they control their stride variables from stride-to-stride, then changing the control gains in the model should change the fluctuation dynamics of the model's stride variables in the same ways as observed experimentally between $\mathrm{YH}$ and $\mathrm{OH}$ adults.

\section{Results}

On average, older healthy $(\mathrm{OH})$ adults walked with slightly shorter $(p=0.021)$ and faster $(p<0.001)$ strides than young healthy $(\mathrm{YH})$ adults at each speed (Fig. 1A). However, there were no significant differences between groups $(p=0.569)$ for average stride speeds (Fig. 1A). Across all speeds, $\mathrm{OH}$ adults exhibited greater variability (Fig. $1 \mathrm{~B})$ for stride times $(p=0.001)$, lengths $(\mathrm{p}=0.036)$ and speeds $(\mathrm{p}<0.001)$.

Both groups exhibited much greater relative variability along the GEM than perpendicular to it (Fig. 2B; $p_{\text {Dir }}<<0.0001$ ). However, there were no significant differences either across walking speeds $\left(\mathrm{p}_{\mathrm{Spd}}=0.970\right)$, or between $\mathrm{YH}$ and $\mathrm{OH}$ adults $\left(\mathrm{p}_{\mathrm{Grp}}=0.520\right)$.

Both groups exhibited strong statistical persistence $(\alpha>>1 / 2)$ for both stride times $\left(T_{n}\right)$ and stride lengths $\left(L_{n}\right)$, but anti-persistence $(\alpha<1 / 2)$ for stride speeds $\left(S_{n}\right)$ (Fig. 3B). There were small, but statistically significant differences between speeds only for $\alpha\left(L_{n}\right)$ $\left(p_{\text {spd }}=0.038\right)$. However, there were no significant differences between $\mathrm{YH}$ and $\mathrm{OH}$ adults for any primary stride parameters (Fig. 3B; all $\mathrm{p}_{\mathrm{Grp}}>0.560$ ). With respect to the Speed GEM (Fig. 3C), differences in statistical persistence between $\delta_{T}$ and $\delta_{P}$ directions were highly statistically significant $\left(\mathrm{p}_{\text {Dir }}<<0.0001\right.$ ), with all participants exhibiting strong statistical persistence in the $\delta_{T}$ direction and slight anti-persistence in the $\delta_{P}$ direction, as expected [24-26]. However, there were no significant differences 
A
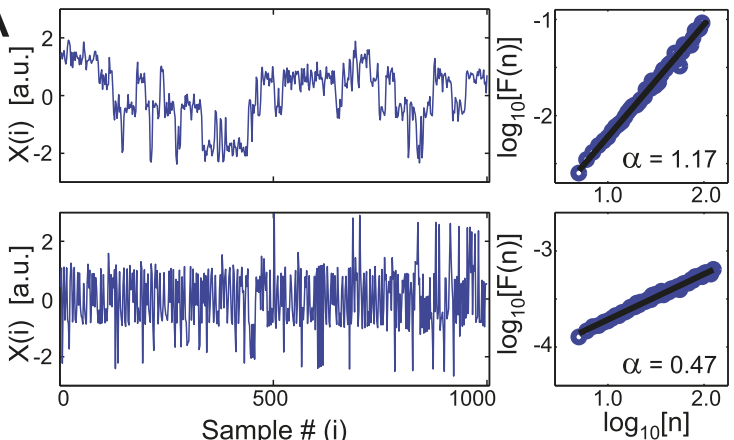

B
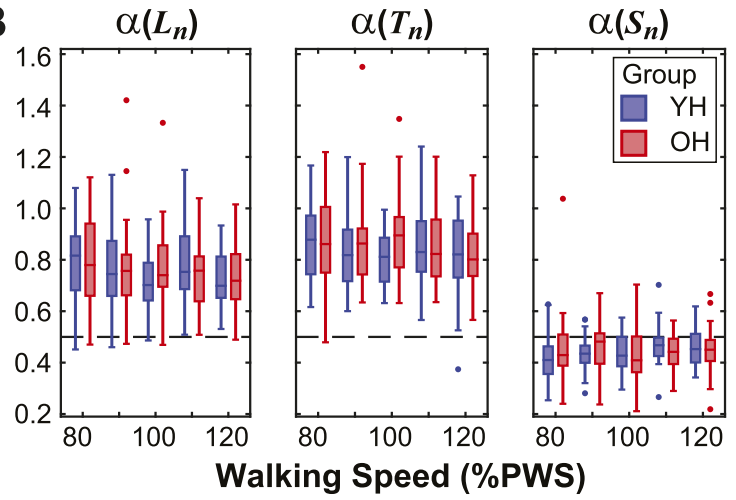

C

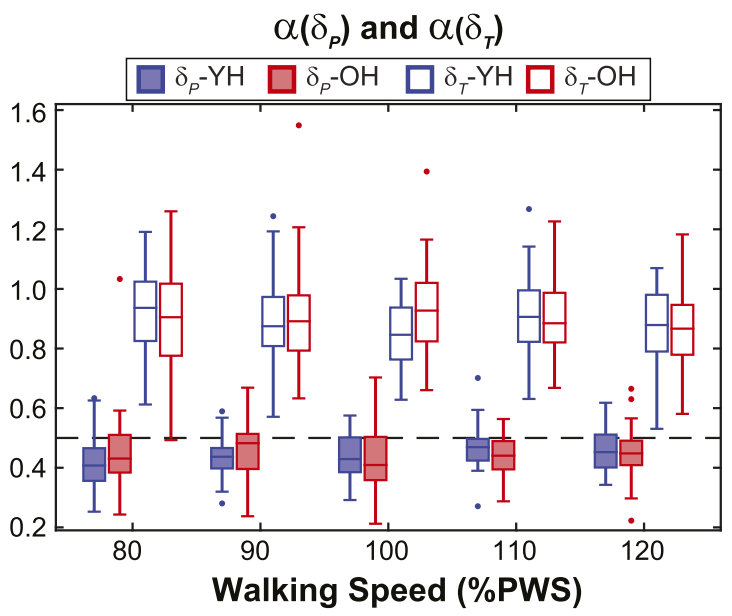

Fig. 3. Stride-To-Stride Control: $\boldsymbol{A}$ : Schematic showing example calculation of DFA $\alpha$ exponents. Each time series shown has a standard deviation of 1 . The strongly persistent time series (top) is consistent with being weakly controlled and yields a large value of $\alpha \gg 1 / 2$. The approximately uncorrelated signal (bottom) is consistent with being strongly controlled and yields a much smaller value of $\alpha$ $\approx 1 / 2$. B: Boxplots of DFA $\alpha$ exponents for $L_{n}, T_{n}$, and $S_{n}$ for both $\mathrm{YH}$ and $\mathrm{OH}$ groups walking at all 5 speeds. Differences between speeds were statistically significant for $L_{n}\left(\mathrm{p}_{\mathrm{Spd}}=0.038\right)$, but not for $T_{n}\left(\mathrm{p}_{\mathrm{Spd}}=0.214\right)$ or $S_{n}\left(\mathrm{p}_{\mathrm{Spd}}=0.498\right)$. There were no significant differences between $\mathrm{OH}$ and $\mathrm{YH}$ groups for any parameter: $L_{n}$ $\left(\mathrm{p}_{\mathrm{Grp}}=0.785\right), T_{n}\left(\mathrm{p}_{\mathrm{Grp}}=0.564\right)$ or $S_{n}\left(\mathrm{p}_{\mathrm{Grp}}=0.982\right)$. $\mathrm{C}$ : Boxplots of DFA $\alpha$ exponents for $\delta_{P}$ and $\delta_{T}$ for both $\mathrm{YH}$ and $\mathrm{OH}$ groups walking at all 5 speeds. Differences between $\delta_{P}$ and $\delta_{T}$ directions were highly statistically significant $\left(\mathrm{p}_{\text {Dir }}<<0.0001\right)$. However there were no significant differences either across speeds ( $\left.\mathrm{p}_{\mathrm{Spd}}=0.137\right)$, or between $\mathrm{YH}$ and $\mathrm{OH}$ groups $\left(\mathrm{p}_{\mathrm{Grp}}=0.863\right)$. Thus, these healthy older adults $(\mathrm{OH})$ appear to correct deviations in their stride-to-stride fluctuations in the same way and to the same degree as younger $(\mathrm{YH})$ participants. $\operatorname{In}(\boldsymbol{B})$ and $(\boldsymbol{C})$, box plots were constructed in the same manner as in Fig. 1.

either across speeds ( $\left.\mathrm{p}_{\mathrm{Spd}}=0.137\right)$, or between $\mathrm{YH}$ and $\mathrm{OH}$ adults ( $\left.\mathrm{p}_{\mathrm{Grp}}=0.863\right)$.

Parameter sensitivity studies carried out using simulations of our computational model (Fig. 4) demonstrated that varying additive noise increased variability in all stride variables (Fig. 4A), but did not change their statistical persistence/anti-persistence
(Fig. 4B). Varying multiplicative noise yielded little change in variability (Fig. 4C) and no changes in statistical persistence/antipersistence (Fig. 4D). Conversely, independently varying the controller gain systematically altered both the variability (Fig. 4E) and statistical persistence/anti-persistence (Fig. 4F) for all of the relevant variables. Thus, varying the noise inputs to the model without changing the control altered the variability of stride variables, but not their DFA $\alpha$ exponents. Conversely, varying only the strength of stride-to-stride regulation in the model (independent of any changes in noise) was the only manipulation that yielded altered DFA $\alpha$ exponents of the resulting stride variables.

\section{Discussion}

Gait variability is an important indicator of overall walking function $[7,14]$ and fall risk $[8,9,18]$ for older individuals. Therefore, it is important to better understand where gait variability comes from and what factors contribute to that variability [22]. In particular, there is a need to distinguish between variability that arises simply from changes in neuromotor noise [10-13] from that which may arise from changes in how people regulate their stride variables (e.g., $L, T$, etc.) from each stride to the next [23-25].

The present study tested $\mathrm{OH}$ adults who walked at similar preferred speeds as $\mathrm{YH}$ adults (Table 1), suggesting that they were indeed quite healthy [32]. Thus, increased gait variability occurs (Fig. 1B) even in very healthy older adults with very low fall risk. We previously found these variability increases were independent of changes in walking speed [7], but partly explained by decreased lower extremity strength and range of motion [7] and increased muscle activation variability [14]. The present findings extend that work to show that despite increased neuromuscular and kinematic variability, these healthy older adults exhibited no differences in how their stride variability was distributed relative to the GEM (Fig. 2B). More importantly, and independent of any changes in variability, these $\mathrm{OH}$ adults also exhibited no differences in how they regulated (from each stride to the next), either their basic stride variables (Fig. 3B) or deviations relative to the GEM (Fig. 3C).

Using our previously validated computational model [23,24], we could explicitly separate effects of noise from those of control. Specifically, the noise terms $(N \& \eta)$ in Eq. (4) are independent from the control input, $\mathbf{u}\left(\mathbf{x}_{n}\right)$. Thus, in Fig. $4 \boldsymbol{A}-\boldsymbol{D}$ we varied only the noise inputs, with no changes in control. Conversely, in Fig. $4 \boldsymbol{E}-\boldsymbol{F}$, we varied only the strength (gain) of the control input, with no changes in noise. The experimental differences observed between $\mathrm{OH}$ and YH participants (Figs. 1-3) were entirely consistent with the idea that the increased variability exhibited by the $\mathrm{OH}$ adults originated solely from greater physiological neuromotor noise [10-13] (Fig. 4A-B), with no changes in their capacity to regulate strideto-stride fluctuations in their stride variables across consecutive strides (Fig. 4E-F). All results indicate that these $\mathrm{OH}$ adults implemented the same underlying stride-to-stride control strategy that the YH adults did. This suggests these very healthy older adults were just as successful at reducing the effects of input noise (as quantified by the methods applied here) as young adults. The older adults just started with more physiological noise [10-13] to begin with.

Although our $\mathrm{OH}$ adults did not exhibit differences in how they regulated their stepping movements from stride-to-stride, they did walk differently than the YH adults. In particular, in addition to their greater overall variability (Fig. 1A), they also took slightly shorter/faster strides to achieve the same walking speeds (Fig. 1A). This may suggest that these $\mathrm{OH}$ adults might have adopted, possibly in response to their greater physiological noise, these slightly shorter/faster strides as a general strategy to allow them to continue to employ the same stride-to-stride error-correction strategy as their YH adult counterparts. 

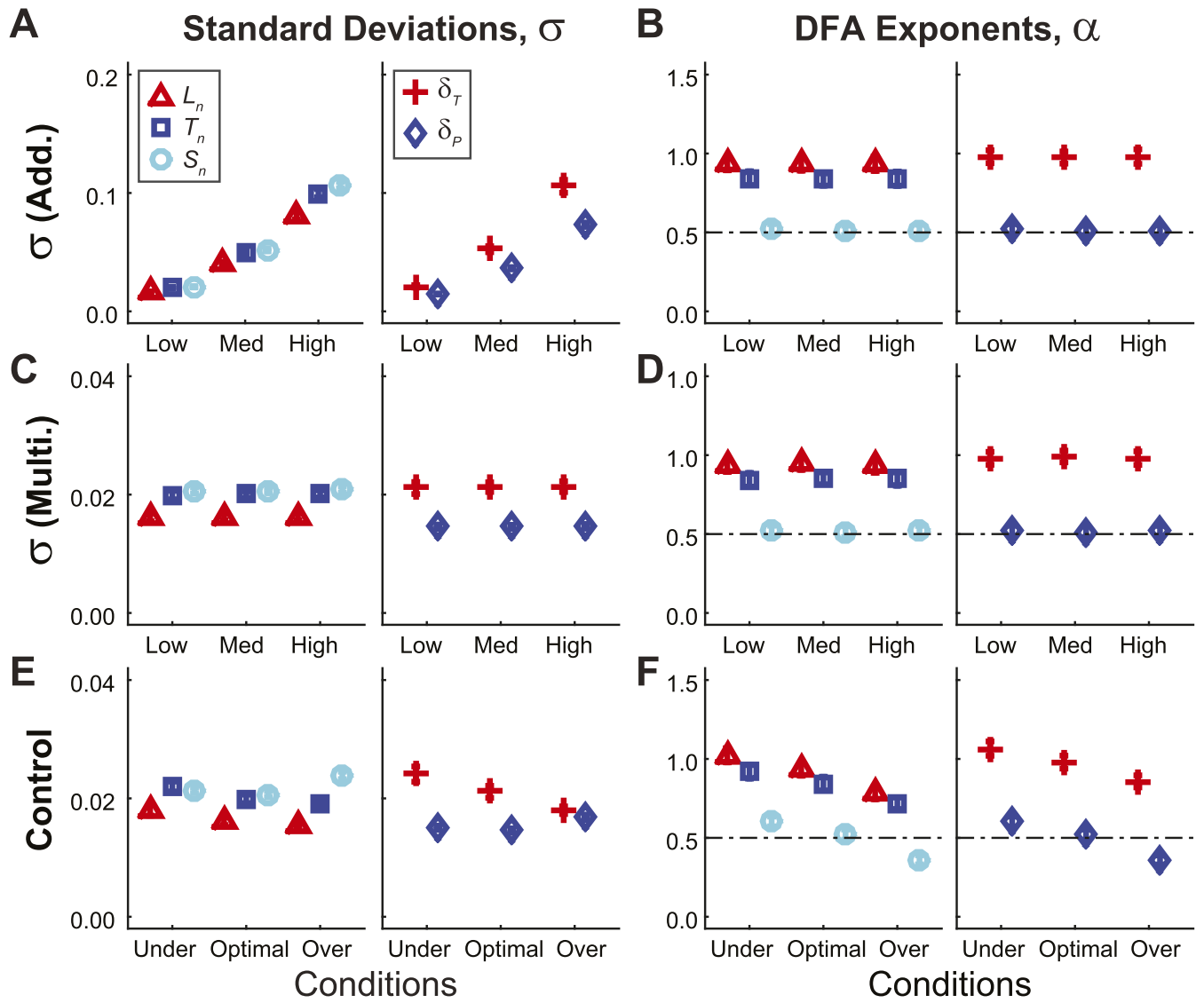

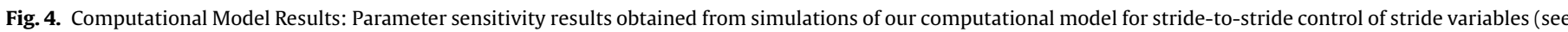

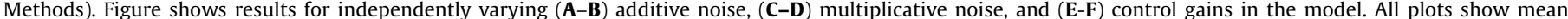

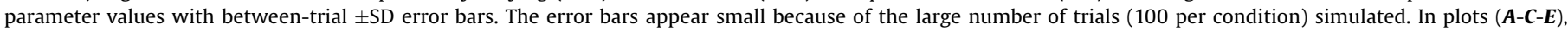

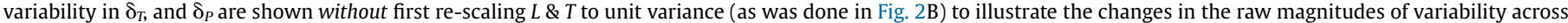

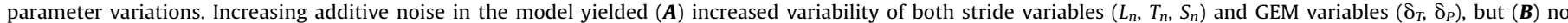

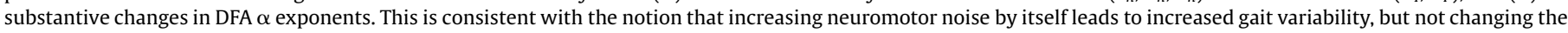

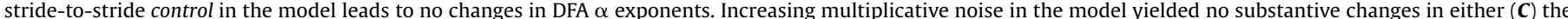

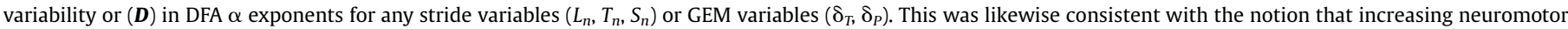

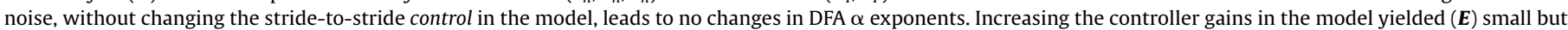

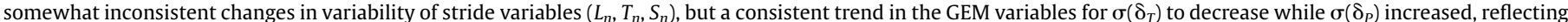

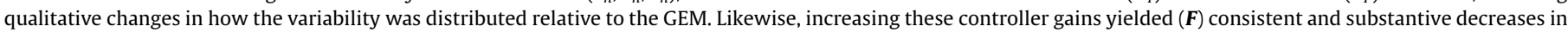

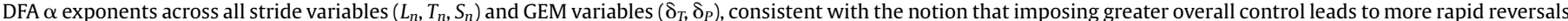

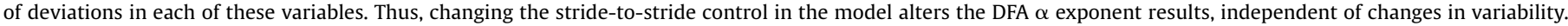

In a previous study, Decker et al. [26], also applied these same analyses to compare young and older adults. They did not report results for overall variability magnitude. However, unlike our findings, their older adults did shift how their variability was distributed relative to the constant-speed GEM, exhibiting greater relative variance in the $\delta_{P}$ direction and less in the $\delta_{T}$ direction. Similar to our findings though (Fig. 3C), Decker et al. also found no significant group differences in statistical persistence (DFA $\alpha$ ) for stride-to-stride fluctuations in either $\delta_{T}$ or $\delta_{p}$. Their older adults walked at significantly slower preferred speeds $(0.77 \pm 0.04 \mathrm{~m} / \mathrm{s})$ than their younger cohort $(1.08 \pm 0.04 \mathrm{~m} / \mathrm{s})$ and Decker et al. neither controlled for walking speed experimentally, nor adjusted for speed differences (e.g., as a covariate) in their analyses. However, our finding that the variables of interest here were not sensitive to changes in walking speed in either group (Figs. Fig. 2B and Fig. 3B-C), suggests that the group differences reported by Decker et al. were not likely caused by differences in walking speed. However, slow walking speed is also a strong predictor of overall health [32], suggesting that their older adults were generally less healthy than those tested here. Such differences in overall health status are most likely the primary source of differences between their findings and ours. Thus, we believe these two independent experimental results to be both consistent and complimentary.

The findings of the present study demonstrate that healthy older adults experience modest increases in neuromotor noise, but can accommodate/compensate for this added noise without sacrificing their preferred stride-to-stride control strategy. Thus, age-related increases in gait variability likely precede deterioration of stepping control: i.e., of how older adults regulate their stride variables from stride-to-stride. With more deteriorated function however (e.g., as in [26]), older adults' ability to continue employing these stepping strategies successfully may start to degrade. These findings are consistent with, and supported by, our previous model predictions, which exhibited similar trends with regard to fall risk [18].

\section{Conflict of interest statement}

The authors declare that there are no conflicts of interest associated with this work. 


\section{Acknowledgements}

Partial funding provided by Biomedical Engineering Research Grant \# RG-02-0354 from the Whitaker Foundation (to JBD), National Science foundation (NSF) Grant \# 0625764 (to JPC), and National Institutes of Health (NIH) Grant 1-R01-AG049735 (to JBD \& JPC). The authors thank Dr. Hyun Gu Kang and Dr. Deanna H. Gates for their assistance with data collection and initial processing.

\section{References}

[1] S.N. Robinovitch, F. Feldman, Y. Yang, R. Schonnop, P.M. Leung, T. Sarraf, et al. Video capture of the circumstances of falls in elderly people residing in longterm care: an observational study, Lancet 381 (2013) 47-54.

[2] P. Kannus, H. Sievänen, M. Palvanen, T. Järvinen, J. Parkkari, Prevention of falls and consequent injuries in elderly people, Lancet 366 (2005) 1885-1893.

[3] J.A. Stevens, P.S. Corso, E.A. Finkelstein, T.R. Miller, The costs of fatal and nonfatal falls among older adults, Inj. Prev. 12 (2006) 290-295.

[4] D.A. Sterling, J.A. O'Connor, J. Bonadies, Geriatric falls: injury severity is high and disproportionate to mechanism, J. Trauma 50 (2001) 116-119.

[5] J.A. Stevens, K.A. Mack, L.J. Paulozzi, M.F. Ballesteros, Self-Reported falls and fall-Related injuries among persons Aged $\geq 65$ years-United States, 2006, J. Safety Res. 39 (2008) 345-349.

[6] N. Niino, S. Tsuzuku, F. Ando, H. Shimokata, Frequencies and circumstances of falls in the national institute for longevity sciences, longitudinal study of aging (NILS-LSA), J. Epidemiol. 10 (2000) S90-S94.

[7] H.G. Kang, J.B. Dingwell, Separating the effects of age and speed on gait variability during treadmill walking, Gait Posture 27 (2008) 572-577.

[8] M.J.P. Toebes, M.J.M. Hoozemans, R. Furrer, J. Dekker, J.H. van Dieën, Local dynamic stability and variability of gait are associated with fall history in elderly subjects, Gait Posture 36 (2012) 527-531.

[9] J. Verghese, R. Holtzer, R.B. Lipton, C. Wang, Quantitative gait markers and incident fall risk in older adults, J. Gerontol. Ser. A: Biol. Sci. Med. Sci. 64A (2009) 896-901.

[10] R.M. Enoka, E.A. Christou, S.K. Hunter, K.W. Kornatz, J.G. Semmler, A.M. Taylor et al., Mechanisms that contribute to differences in motor performance between young and old adults, J. Electromyogr. Kinesiol. 13 (2003) 1-12.

[11] S.W. Shaffer, A.L. Harrison, Aging of the somatosensory system: a translationa perspective, Phys. Ther. 87 (2007) 193-207.

[12] E.A. Christou, Aging and variability of voluntary contractions, Exerc. Sport Sci. Rev. 39 (2011) 77-84.

[13] P. Aagaard, C. Suetta, P. Caserotti, S.P. Magnusson, M. Kjær, Role of the nervous system in sarcopenia and muscle atrophy with aging: strength training as a countermeasure, Scand. J. Med. Sci. Sports 20 (2010) 49-64.
[14] H.G. Kang, J.B. Dingwell, Dynamics and stability of muscle activations during walking in healthy young and older adults, J. Biomech. 42 (2009) 2231-2237.

[15] J.S. Brach, S. Studenski, S. Perera, J.M. VanSwearingen, A.B. Newman, Stance time and step width variability have unique contributing impairments in older persons, Gait Posture 27 (2008) 431-439.

[16] A. Mirelman, T. Herman, M. Brozgol, M. Dorfman, E. Sprecher, A. Schweiger, et al., Executive function and falls in older adults: new findings from a fiveYear prospective study link fall risk to cognition, PLoS One 7 (2012) e40297.

[17] H.G. Kang, J.B. Dingwell, The effects of walking speed, strength and range of motion on gait stability in healthy older adults, J. Biomech. 41 (2008) 2899-2905.

[18] PE Roos, JB. Dingwell, Influence of simulated neuromuscular noise on movement variability and fall risk in a 3D dynamic walking model, J. Biomech. 43 (2010) 2929-2935.

[19] J.P. Cusumano, P. Cesari, Body-Goal variability mapping in an aiming task, Biol. Cybern. 94 (2006) 367-379.

[20] E. Todorov, Optimality principles in sensorimotor control, Nat. Neurosci. 7 (2004) 907-915.

[21] J.P. Cusumano, J.B. Dingwell, Movement variability near goal equivalent manifolds: fluctuations, control, and model-Based analysis, Hum. Mov. Sci. 32 (2013) 899-923.

[22] J. John, J.B. Dingwell, J.P. Cusumano, Error correction and the structure of interTrial fluctuations in a redundant movement task, PLoS Comput. Biol. 12 (2016) e1005118.

[23] J.B. Dingwell, J.P. Cusumano, Identifying stride-To-Stride control strategies in human treadmill walking, PLoS One 10 (2015) e0124879.

[24] J.B. Dingwell, J. John, J.P. Cusumano, Do humans optimally exploit redundancy to control step variability in walking? PLoS Comput. Biol. 6 (2010) e1000856.

[25] J.B. Dingwell, J.P. Cusumano, Re-Interpreting detrended fluctuation analyses of stride-To-Stride variability in human walking, Gait Posture 32 (2010) 348-353.

[26] L.M. Decker, F. Cignetti, J.F. Potter, S.A. Studenski, N. Stergiou, Use of motor abundance in young and older adults during dual-Task treadmill walking, PLoS One 7 (2012) e41306.

[27] P. Terrier, O. Dériaz, Persistent and anti-persistent pattern in stride-to-stride variability of treadmill walking: influence of rhythmic auditory cueing, Hum. Mov. Sci. 31 (2012) 1585-1597.

[28] M. Roerdink, A. Daffertshofer, V. Marmelat, P.J. Beek, How to sync to the beat of a persistent fractal metronome without falling off the treadmill? PLoS One 10 (2015) e0134148.

[29] J.B. Dingwell, L.C. Marin, Kinematic variability and local dynamic stability of upper body motions when walking at different speeds, J. Biomech. 39 (2006) 444-452.

[30] J.M. Hausdorff, Gait dynamics, fractals and falls: finding meaning in the strideto-stride fluctuations of human walking, Hum. Mov. Sci. 26 (2007) 555-589.

[31] C.M. Harris, D.M. Wolpert, Signal-Dependent noise determines motor planning, Nature 394 (1998) 780-784.

[32] S. Studenski, S. Perera, K. Patel, C. Rosano, K. Faulkner, M. Inzitari, et al., Gait speed and survival in older adults, JAMA 305 (2011) 50-58. 